Conclusion: The trial is challenging, both in terms of recruitment, and motivating behaviour change. The presentation will elaborate on the recruitment experience into a trial evaluating the effectiveness of interventions in ethnic minority populations.

\section{UNDER-REPORTING OF TOBACCO USE AMONG BANGLADESH WOMEN IN ENGLAND; A CROSS-SECTIONAL STUDY}

M Roth, A Aitsi-Selmi, H Wardle, J Mindell. Department of Epidemiology and Public Health, University College London, London, UK

\section{doi:10.1136/jech.2009.096735}

Objective: To investigate the prevalence of under-reported use of tobacco among Bangladeshi women and the characteristics of this group.

Design: Cross-sectional surveys.

Setting: Private households in England.

Participants: 996 Bangladeshi women aged 16 years and above, 302 with a valid saliva sample and 694 without, in the 1999 and 2004 Health Surveys for England.

Main Outcome Measure: Prevalence of under-reported tobacco use (estimated using self-reported tobacco use and cotinine level from a saliva sample). Predictors of tobacco use status: self-reported user; cotinine-validated non-user; or under-reporting user.

Results: $15 \%$ of Bangladeshi women with a saliva sample underreported their personal tobacco use. Under-reporting users were similar to self-reported users in terms of socio-demographic, socioeconomic, and tobacco-related variables, except for being much more likely to report chewing paan (a mixture of betel leaf, lime and areca nut) without tobacco (47\% vs. 9\%, p<0.001). Underreporters differed significantly from cotinine-validated non-users in most respects, including age, birth country, education level, level of spoken English, language of the interview, chewing paan without tobacco, and presence of relatives in the interview. Regression analyses confirmed that under-reporters did not differ significantly from self-reported users regarding age, education level, or exposure to passive smoking. Under-reporters were generally older and less likely to be educated above $O$ level compared with cotinine-validated non-users. Both self-reported users (odds ratio $0.11,95 \%$ CI 0.04 to 0.30 ) and cotinine-validated non-users (odds ratio $0.42,95 \%$ CI 0.20 to 0.89 ) were far less likely to report chewing paan without tobacco compared with underreporters.

Conclusion: Contrary to our a priori hypothesis, under-reporters were not British-born, English-speaking young women likely to be concealing smoking but resembled self-reported tobacco users except for being much more likely to report chewing paan without tobacco. Further investigation is needed to discover whether the under-reporting was concealment or a lack of awareness that the paan they chewed contained tobacco.

\section{Plenary session}

\section{ARE POOR MOTHERS AND THEIR INFANTS HEALTHIER IN RICHER AREAS? THE PROTECTIVE EFFECT OF AREA SOCIO- ECONOMIC DENSITY}

${ }^{1} \mathrm{C}$ Albor, ${ }^{1} \mathrm{~K}$ Pickett, ${ }^{2} \mathrm{RG}$ Wilkinson, ${ }^{3} \mathrm{D}$ Ballas. ${ }^{1}$ Department of Health Sciences, University of York, York, UK; ${ }^{2}$ Division of Epidemiology and Community Health, University of Nottingham Medical School, Nottingham, UK; ${ }^{3}$ Department of Geography, University of Sheffield, Sheffield, UK

\section{doi:10.1136/jech.2009.096735m}

Objective: To examine whether poor mothers and their infants have better or worse health when they live in affluent neighbourhoods.
Design: Cross-sectional analysis of 14465 white mothers surveyed in the first wave of the Millennium Cohort Study, 3654 of these mothers were defined as poor. The socio-economic context for poor mothers was measured by lower super output area (LSOA)-level measures of income. UK-wide analyses used the LSOA decile ranks of the index of multiple deprivation (IMD) income domain. A subset England-only analysis of 7288 mothers used continuous IMD income domain scores.

Outcome Measures: Maternal self-rated health and limiting longterm illness (LLI), low birthweight (LBW), and preterm delivery. Logistic regression models were run separately for poor $(<£ 10400)$ and non-poor households. Models were adjusted for age, marital status, parity, urban status, duration at address, occupational class and educational attainment.

Results: For poor mothers, odds for LBW and LLI increased with every decile of area income, by $9 \%$ and $8 \%$ respectively $(p<0.05$ all models). This contrasted with findings for non-poor mothers - for whom odds decreased $6 \%$ and $5 \%$ respectively (not significant for LLI). In the subset England-only analysis, for poor mothers, area income was positively associated with LLI and LBW but significant only for LLI. Self-rated health did not vary significantly across areas for poor mothers. In contrast, for non-poor mothers, poor/fair self-rated health was negatively associated with arealevel income, decreasing by $11 \%$ for each area income decile $(p<0.001$ all models and England subset). Odds of preterm delivery decreased for poor mothers by $7 \%$ per decile $(p<0.05)$, but this was not significant for England-only analyses. No models examining area income in relation to preterm delivery were significant for non-poor mothers.

Conclusion: Apart from preterm delivery, health outcomes of nonpoor mothers are improved when they live in more affluent areas. This is not the case for poor mothers, who do not have better selfrated health, and who have higher risk of LBW and LLI in richer areas. These findings may support a psycho-social causal model mediated by area socio-economic density. Further work is needed to test mediating pathways such as social engagement and class discrimination.

\section{SECONDHAND SMOKE EXPOSURE ASSESSED USING SERUM COTININE: ASSOCIATIONS WITH MYOCARDIAL INFARCTION STROKE AND CARDIOVASCULAR RISK FACTORS IN ADULT MEN AND WOMEN}

${ }^{1} \mathrm{BJ}$ Jefferis, ${ }^{2} \mathrm{GDO}$ Lowe, ${ }^{2} \mathrm{P}$ Welsh, ${ }^{3} \mathrm{DA}$ Lawlor, ${ }^{4} \mathrm{~S}$ Ebrahim, ${ }^{1} \mathrm{SG}$ Wannamethee, ${ }^{5}$ DG Cook, ${ }^{5} \mathrm{PH}$ Whincup. ${ }^{1}$ British Regional Heart Study, Department of Primary Care and Population Health, UCL Medical School, London, UK; ${ }^{2}$ Division of Cardiovascular and Medical Sciences, University of Glasgow, Royal Infirmary, 10 Alexandra Parade, Glasgow, UK: ${ }^{3}$ MRC CAiTE Centre, University of Bristol, Oakfield House, Oakfield Grove, Bristol, UK; ${ }^{4}$ Non-Communicable Diseases Epidemiology Unit, Department of Epidemiology and Population Health, London School of Hygiene and Tropical Medicine, Keppel Street, London, UK; ${ }^{5}$ Division of Community Health Sciences, St George's, University of London, Cranmer Terrace, London, UK

doi:10.1136/jech.2009.096735n

Objectives: Second-hand smoke (SHS) exposure is associated with elevated CVD risks. Previous studies have implicated altered platelet activity or endothelial dysfunction and changes in circulating levels of $\mathrm{HDL}$, homocysteine and inflammatory markers. However most studies have imprecise exposure measurements and the mechanism remains uncertain. Therefore we examine associations between cotinine, a circulating biochemical marker of SHS exposure, and CVD risk factors, incident CHD and stroke in non-smoking men and women.

Methods: 4252 men and 4286 women aged 60-79 years in parallel prospective population-based studies assessed in Primary Care centres in 25 British towns in 1998-2000, with median 7.7 year follow-up for fatal and non-fatal MI $(n=445)$ and stroke $(n=386)$. Medical history, health behaviours and demographic data were reported in questionnaires and nurses recorded an ECG, made anthropometric measurements and took fasting blood samples 